\title{
Fratura toracolombar tipo explosão: comparação do tratamento conservador em pacientes com e sem fratura do arco vertebral posterior ${ }^{*}$
}

\author{
Thoracolumbar burst fracture: comparing conservative \\ treatment in patients with and without fracture \\ of the posterior vertebral arch
}

\author{
Osmar Avanzi ${ }^{1}$, Élcio Landim ${ }^{2}$, Robert Meves ${ }^{3}$, Maria Fernanda Silber Caffaro ${ }^{4}$, \\ Rodrigo Rezende ${ }^{5}$, Murilo Tavares Daher ${ }^{6}$, Bruno César Aprile ${ }^{6}$
}

\section{RESUMO}

Objetivo: Comparar clínica e radiologicamente os resultados a longo prazo do tratamento conservador da fratura explosão toracolombar, em pacientes com e sem fratura do arco vertebral posterior, com o propósito de avaliar eventuais diferenças na evolução destes dois tipos de lesão. Métodos: Foram avaliados, retrospectivamente, os prontuários e exames de imagem (radiografias e tomografias computadorizadas) de 25 pacientes sem déficit neurológico, com fratura toracolombar tipo explosão tratados não cirurgicamente e comparados o grau de progressão da cifose entre os casos com fratura da lâmina (grupo 1) e sem fratura posterior (grupo 2). Desses, 13 pacientes foram submetidos à avaliação comparativa por meio da escala visual analógica de dor (VAS), da escala de dor e trabalho de Denis e do questionário de qualidade de vida SF-36. Resultados: Foram analisados 25 pacientes (36\% do grupo A e $74 \%$ do grupo B) com tempo médio de seguimento de 111,64 meses. Não houve diferença em relação ao grau de progressão da cifose durante o seguimento entre os grupos $A$ e $B\left(5,22^{\circ} \times 4,63^{\circ}-p\right.$ $=0,650)$. Dos 13 pacientes analisados funcionalmente, $46 \%$ eram do grupo A e $54 \%$ do grupo $B$. Nesta avaliação, apesar da VAS pior $(1,83 \times 5,00-p=0,015)$
* Trabalho realizado no Grupo de Coluna do Departamento de Ortopedia e Traumatologia da Faculdade de Ciências Médicas da Santa Casa de Misericórdia de São Paulo (DOT-FCMSCSP) Pavilhão "Fernandinho Simonsen" - São Paulo (SP), Brasil.

1. Doutor, Professor Adjunto, Consultor do Grupo da Coluna do Departamento de Ortopedia e Traumatologia da Faculdade de Ciências Médicas da Santa Casa de Misericórdia de São Paulo FCMSCSP - São Paulo (SP), Brasil.

2. Professor, Chefe do Grupo da Coluna do Departamento de Ortopedia e Traumatologia da Faculdade de Ciências Médicas da Santa Casa de Misericórdia de São Paulo - FCMSCSP - São Paulo (SP), Brasil.

3. Professor Assistente do Grupo da Coluna do Departamento de Ortopedia e Traumatologia da Faculdade de Ciências Médicas da Santa Casa de Misericórdia de São Paulo - FCMSCSP - São Paulo (SP), Brasil.
4. Mestre, Assistente do Grupo da Coluna do do Departamento de Ortopedia e Traumatologia da Faculdade de Ciências Médicas da Santa Casa de Misericórdia de São Paulo - FCMSCSP - São Paulo (SP), Brasil.

5. Pós-graduando do Departamento de Ortopedia e Traumatologia da Faculdade de Ciências Médicas da Santa Casa de Misericórdia de São Paulo - FCMSCSP - São Paulo (SP), Brasil.

6. Residente do 3o ano do Departamento de Ortopedia e Traumatologia da Faculdade de Ciências Médicas da Santa Casa de Misericórdia de São Paulo - FCMSCSP - São Paulo (SP), Brasil.

Endereço para correspondência: Bruno César Aprile, Rua Meruoca, 125 - 01547-150 - São Paulo (SP), Brasil. Tels.: (11) 61615191/9145-1525.

E-mail: brunoaprile@terra.com.br/robertmeves@hotmail.com Recebido em 11/3/08. Aprovado para publicação em 19/6/08. Copyright RBO2008 
nos pacientes sem fratura posterior (grupo B), não houve diferença em relação à escala de Denis $(4,00$ x 5,71 - p > 0,05) e SF-36 $(98,60$ x 90,83 - p = 0,168) entre os dois grupos. Conclusão: A fratura do arco posterior, isoladamente, parece não ser indicativo de instabilidade ou de mau prognóstico nas fraturas toracolombares tipo explosão.

Descritores - Fraturas da coluna vertebral/terapia; Fraturas da coluna vertebral/radiografia; Canal vertebral; Tomografia computadorizada por raios X; Resultado de tratamento; Estudos retrospectivos

\section{ABSTRACT}

Objective: To make a clinical and radiological comparison of long term results of the conservative treatment of thoracolumbar burst fractures, in patients with and without fracture in the posterior vertebral arch, in order to assess possible differences in the evolution of the two types of lesion. Methods: A retrospective analysis was made of the clinical records and imaging exams (X-rays CT scans) of 25 patients without neurological deficit with thoracolumbar burst fractures treated without surgery, and a comparison was made of the degree of progression of kyphosis between the cases with fracture of the lamina (Group 1) and those without posterior fracture (Group 2). 13 of these patients were submitted to comparative evaluation using the visual analogical scale of pain (VAS), the Denis pain and work scale, and the life quality questionnaire SF-36. Results: 25 patients were analyzed (36\% in group A and $74 \%$ in group B) with mean follow-up of 111.64 months. There was no difference in the degree of kyphosis progression during follow-up between groups $A$ and $B\left(5.22^{\circ} \times 4.63^{\circ}-p=0.650\right)$. Of the 13 patients functionally analyzed, $46 \%$ were from group $A$ and $54 \%$ from group B. In this assessment, despite the worse VAS $(1.83 \times 5.00-p=0.015)$ in patients without posterior fracture (Group B), there was no difference in the Denis scale $(4.00 \times 5.71-p>0.05)$ and SF-36 (98.60 $90.83-p=0.168)$ between the two groups. Conclusion: Taken separately, the posterior arch fracture does not seem to be an indicator of instability or of poor prognosis in thoracolumbar burst fractures.

Keywords - Spinal fractures/therapy; Spinal fractures/ radiography; Spinal canal; Tomography, X-ray computed; Treatment outcome; Retrospective studies

\section{INTRODUÇÃO}

O termo fratura tipo explosão foi descrito por Holdsworth $^{(1)}$. Este autor baseia-se na teoria das duas colunas e considera essas fraturas como lesões estáveis. Whitesides, baseando-se na teoria das duas colunas, as diferenciou em estáveis e instáveis, quando havia lesão dos elementos posteriores: pedículos, lâminas, processos espinhosos e ligamentos interlaminares ${ }^{(2)}$.

Denis, em 1983, introduziu o conceito de três colunas e definiu a fratura explosão como aquela que ocorre por falha das colunas anterior e média a partir de uma força em compressão. Também cita a lesão do arco posterior a partir de carga axial como tipo de fratura em galho verde da lâmina ${ }^{(3)}$. McAfee et al enfatizaram que a fratura da coluna posterior evidenciada na tomografia axial computadoriza (TAC) é fator indicativo de instabilidade ${ }^{(4-5)}$. Apesar do desenvolvimento de estudos biomecânicos e de novos sistemas de instrumentação, estudos clínicos ainda discutem a melhor opção de tratamento para esses pacientes, principalmente quando não há lesão neurológica ${ }^{(6-7)}$.

Alguns autores defendem o tratamento operatório, relatando melhor correção da cifose, possibilidade de descompressão do canal vertebral, prevenção de deterioração neurológica e menor tempo de repouso ${ }^{(8-11)}$. Além disso, a lesão oculta da dura-máter não diagnosticada nos pacientes com fratura de lâmina pode ser fator complicador do tratamento quando não reparada cirurgicamente $^{(12-14)}$. Vários autores indicam o tratamento cirúrgico nos casos com lesão esquelética posterior, mesmo nos pacientes sem lesão neurológica ${ }^{(7,13)}$. Outros, em contrapartida, referem que o tratamento conservador é mais barato e apresenta capacidade similar de prevenir a cifose pós-traumática. Além disso, o fragmento projetado para dentro do canal vertebral é reabsorvido com o tempo e a cifose residual parece não ter relação com a sintomatologia do paciente ${ }^{(15-22)}$. 


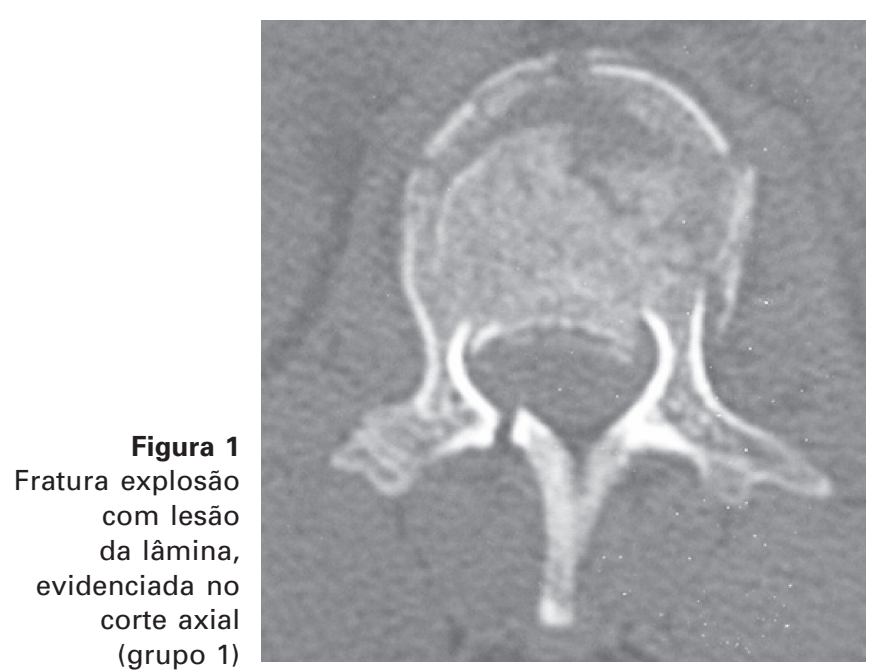

Ademais, a piora neurológica em pacientes neurologicamente normais é desfecho clínico raro.

O objetivo deste estudo é avaliar de forma retrospectiva os resultados clínicos e radiográficos dos pacientes com fratura toracolombar tipo explosão tratados sem cirurgia no DOT-FCMSCSP, comparando o grupo com o sem fratura da lâmina em compressão.

\section{MÉTODOS}

Após aprovação do Comitê de Ética em Pesquisa do DOT-FCMSCSP, foram levantados os prontuários, radiografias e tomografias computadorizadas (TC) do Serviço de Arquivo Médico e Estatística (SAME) referentes aos pacientes portadores de fratura toracolombar tipo explosão tratados conservadoramente entre janeiro de 1992 e janeiro de 2005 no Pavilhão Fernandinho Simonsen - Departamento de Ortopedia e Traumatologia da Santa Casa de Misericórdia de São Paulo.

Todos foram tratados com colete de Jewett ou gesso antigravitacional por quatro a seis meses, com deambulação precoce.

Foram incluídos neste estudo os pacientes adultos com fratura tipo explosão segundo a classificação de Denis $^{(3)}$. Todas eram fraturas agudas (menos de 10 dias), comprometiam um único segmento vertebral, não apresentavam déficit neurológico e tinham pelo menos 36 meses de seguimento ${ }^{(3)}$. Foram excluídos os casos: fora do segmento toracolombar (T11-L2), sem documentação completa, lesões que apresentavam

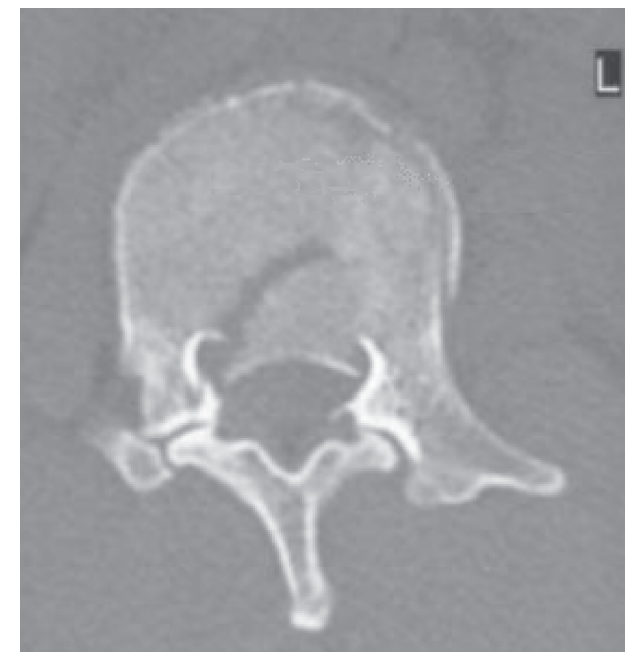

Figura 2

Fratura explosão sem lesão da lâmina (grupo 2)

componente de distração ou rotação, como fratura-luxação facetária ou lesões ligamentares por flexo-distração e também as fraturas patológicas.

A análise radiográfica foi realizada levando em consideração a cifose inicial e a final, medida no último acompanhamento, segundo metodologia proposta por Kuklo et $a l^{(23)}$. Também foram analisados os filmes iniciais das tomografias computadorizadas (TC) através da aferição do comprometimento do canal vertebral (diâmetro sagital médio) ${ }^{(24)}$ e averiguada a presença de fratura da lâmina, dividindo, em consequiência, os pacientes em grupo 1 - com fratura e grupo 2 sem esta lesão (figuras 1 e 2).

Os pacientes que responderam à convocação foram submetidos a análise funcional, baseada no nível de dor segundo a escala visual analógica (VAS) e a escala de dor e trabalho de Denis ${ }^{(3)}$ (tabelas 1 e 2). Os pacien-

TABELA 1

Escala funcional de dor segundo Denis

\begin{tabular}{cl}
\hline Pontuação & \multicolumn{1}{c}{ Critérios da escala de dor } \\
\hline 1 & Sem dor \\
2 & Dor mínima, sem uso de medicação \\
3 & Dor moderada, com uso ocasional medicação \\
4 & Dor moderada a grave, com uso constante de \\
& medicação \\
5 & Dor grave, com uso crônico de medicação
\end{tabular}

Fonte: Traduzido de: Denis F. The three column spine and its significance in the classification of acute thoracolumbar spinal injuries. Spine. 1983;8(8):817-31. 
TABELA 2

Escala funcional de trabalho segundo Denis

\begin{tabular}{|c|c|}
\hline Pontuação & Critérios da escala de trabalho \\
\hline 1 & Retorno ao trabalho pesado \\
\hline 2 & $\begin{array}{l}\text { Retorno ao trabalho sedentário, sem restrição } \\
\text { de peso }\end{array}$ \\
\hline 3 & Retorno ao trabalho, mas mudou de atividade \\
\hline 4 & Retorno ao trabalho, porém por meio período \\
\hline 5 & Incapaz para o trabalho \\
\hline
\end{tabular}

Fonte: Traduzido de: Denis F. The three column spine and its significance in the classification of acute thoracolumbar spinal injuries. Spine. 1983;8(8):817-31.

tes também foram submetidos à aplicação do questionário de qualidade de vida Short-Form 36 (25) (SF-36) no último acompanhamento. A avaliação neurológica foi realizada de acordo com a escala de Frankel et al(26) (tabela 3).

TABELA 3

Classificação de Frankel

\begin{tabular}{cll}
\hline Classificação & Motricidade & Sensibilidade \\
\hline A & ausente & Ausente \\
B & ausente & Presente \\
C & presente não útil & Presente \\
D & presente útil & Presente \\
E & normal & Normal \\
\hline
\end{tabular}

Fonte: Traduzido de: Frankel HL, Hancock DO, Hyslop G, Melzak J, Michaelis LS, Ungar $\mathrm{GH}$, et al. The value of postural reduction in the initial management of closed injuries of the spine with paraplegia and tetraplegia. I. Paraplegia. 1969;7(3):179-92.

Os resultados funcionais e radiográficos foram comparados entre os grupos 1 e 2 e a análise estatística foi realizada com o teste de Mann-Whitney, usando o programa SPSS (Statistical Package for Social Sciensces), versão 13,0. Foi adotado o nível de significância de $5 \%(\mathrm{p}<0,05)$.

\section{RESULTADOS}

Nos pacientes analisados, a média de idade foi de 48,48 anos (com variação de 25 a 75), sendo 13 (52\%) do sexo masculino e 12 (48\%) do sexo feminino; nove $(36 \%)$ apresentavam fratura de lâmina (grupo 1) e em $16(74 \%)$ não se evidenciava essa fratura (grupo 2). O tempo de seguimento médio foi de 111,64 meses (36 a

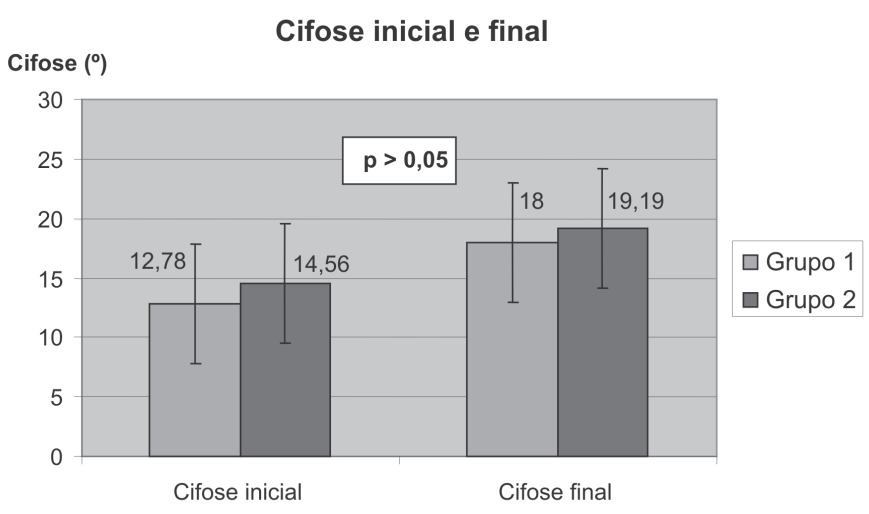

Gráfico 1 - Cifose inicial e final

198); todos apresentaram-se no último seguimento sem déficit neurológico (Frankel E). O mecanismo de trauma mais comum foi a queda de altura (72\%), seguido pelo acidente automobilístico (16\%). O nível mais acometido foi L1 (10 pacientes), seguido por T12 e L2 (nove e seis, respectivamente).

Os grupos analisados foram estatisticamente similares em relação à idade, sexo, tempo de seguimento, comprometimento do canal vertebral, encunhamento do corpo vertebral e grau de cifose inicial.

Quanto à classificação AO, 16 (64\%) eram do tipo A3.1, seis (24\%) tipo A3.2 e três (12\%), A3.3. O grau médio da estenose do canal vertebral foi de $22,7 \%$ (14 a $33 \%$ ) e a perda média da altura do corpo vertebral, de $36,6 \%$ (23 a 50\%). A média da cifose inicial no grupo 1 foi equivalente a $12,78^{\circ}\left(1^{\circ}\right.$ a $\left.30^{\circ}\right)$ e a final, de $18^{\circ}\left(0^{\circ}\right.$ a $\left.32^{\circ}\right)$. No grupo 2 , a média de cifose inicial foi de $14,56^{\circ}\left(4^{\circ}\right.$ a $30^{\circ}$ ) e a final, de $19,19^{\circ}\left(4^{\circ}\right.$ a $\left.45^{\circ}\right)$. A perda em graus foi, em média, de $5,22^{\circ}$ no grupo $1 \mathrm{e}$ de $4,63^{\circ}$ no grupo 2 . Não houve diferença estatística entre os dois grupos $(\mathrm{p}=0,650)$ (gráfico 1).

Do grupo total de pacientes, 13 responderam ao questionário de qualidade de vida SF-36, escala de dor e trabalho de Denis e marcaram o nível de dor no último seguimento segundo a escala visual analógica (VAS). Desses, seis (46\%) eram do grupo 1 e sete $(54 \%)$, do grupo 2. O grupo 1 apresentou valores de VAS estatisticamente melhores do que o grupo $2(1,83 \times 5,00, \mathrm{p}=$ $0,015)$. Houve diferença em relação à escala de dor de Denis entre os dois grupos $(1,50 \times 3,14, p=0,027)$, favorecendo o grupo 1. Quanto à escala de trabalho, não houve diferenças estatisticamente significativas 
entre os grupos $(2,50 \times 2,57, \mathrm{p}=0,826)$. $\mathrm{O}$ mesmo aconteceu quando os valores dor e trabalho foram considerados em conjunto $(4,00 \times 5,71, \mathrm{p}>0,05)$. O grupo 1 apresentou valor médio total do SF-36 de 98,6 ( 84 a 106) e o grupo 2, de 90,83 (83 a 105); também, sem diferença estatística $(\mathrm{p}=0,168)$ (tabela 4$)$.

TABELA 4

Resultados funcionais

\begin{tabular}{lllrrrr}
\hline & & Mínimo & Máximo & Média & $\begin{array}{r}\text { Desvio- } \\
\text { padrão }\end{array}$ & p \\
\hline \multirow{2}{*}{ VAS } & Grupo A & 0 & 8 & 1,83 & 3,13 & 0,015 \\
& Grupo B & 1 & 10 & 5,00 & 3,27 & \\
\hline Denis & Grupo A & 1 & 2 & 1,50 & 0,55 & 0,027 \\
dor & Grupo B & 1 & 5 & 3,14 & 1,35 & \\
\hline Denis & Grupo A & 1 & 5 & 2,50 & 1,52 & 0,826 \\
trabalho & Grupo B & 1 & 4 & 2,57 & 1,13 & \\
\hline SF-36 & Grupo A & 84 & 109 & 98,60 & 11,65 & 0,168 \\
& Grupo B & 83 & 105 & 90,83 & 8,64 & \\
\hline
\end{tabular}

Legenda: VAS = escala visual analógica de dor

\section{DISCUSSÃO}

A fratura tipo explosão após queda de altura e acidente de trânsito é observada freqüentemente em nosso meio, em especial nos pacientes politraumatiza$\operatorname{dos}^{(27)}$.

É reconhecido que o tratamento dessa fratura ainda é controverso, principalmente nos pacientes sem déficit neurológico ${ }^{(15-17,20)}$. Verificamos que a maioria dos estudos não avalia a fratura da lâmina em compressão como critério de instabilidade.

Wood et al, em estudo prospectivo e randomizado, compararam os resultados do tratamento cirúrgico e o conservador em 47 pacientes com fratura toracolombar isolada tipo explosão (24 tratados cirurgicamente e 23 com órtese ou gesso). A análise radiográfica mostrou resultados semelhantes em relação à cifose e comprometimento do canal vertebral. Os resultados clínicos também foram similares, todavia, com gastos e complicações significativamente mais elevados no grupo operado. Entretanto, foram excluídos aqueles com fratura-luxação facetária ou lesão ligamentar por flexo-distração; no entanto, a fratura da lâmina não foi considerada critério de exclusão, nem avaliada separadamente ${ }^{(15)}$.

Prospectivamente, Cantor et al analisaram 18 pacientes com fraturas toracolombares tidas como estáveis (excluíram aqueles com lesão dos elementos posteriores) tratados com órtese em extensão e deambulação precoce ${ }^{(16)}$. Semelhante ao nosso estudo, o tratamento conservador apresentou resultados satisfatórios para a maioria dos casos, contudo, sem incluir aqueles com fratura da lâmina.

Chow et al analisaram retrospectivamente uma série de 24 pacientes com fratura toracolombar instável tipo explosão. Nenhum apresentava fratura de lâmina ou faceta. Dez apresentavam dor, com ou sem alargamento do espaço interespinhoso à palpação. Os pacientes foram tratados com gesso ou órtese em extensão (Jewett), dependendo da gravidade da lesão, e deambulação precoce. Entre esses pacientes, 19 $(79,16 \%)$ apresentavam-se assintomáticos ou com dor mínima a moderada, sem limitações de suas atividades. Os pacientes com aumento do espaço entre os processos espinhosos apresentaram bons resultados em relação à escala de dor e trabalho de Denis. Não foram incluídos os casos com fratura de lâmina, mas esse alargamento interespinhoso poderia representar uma lesão ligamentar posterior ${ }^{(17)}$. A conduta adotada sugere que essa lesão posterior isolada não é critério de indicação cirúrgica.

Tropiano et al, ao analisar retrospectivamente o tratamento conservador em 45 pacientes submetidos a redução fechada e órtese, verificaram que os três que apresentavam lesão ligamentar posterior evoluíram com dor crônica, tendo que ser submetidos à artrodese vertebral. Os 15 pacientes com lesões instáveis decorrentes de lesões ósseas (fratura de lâmina ou faceta) não foram operados e não tiveram seus resultados ressaltados pelos autores, sugerindo valores semelhantes aos da média do grupo geral com fratura toracolombar e lombar tipo explosão(28).

Esses diversos estudos exemplificam bem a controvérsia existente sobre a opção terapêutica nas fraturas toracolombares em explosão.

$\mathrm{Na}$ casuística que analisamos, dos pacientes que foram submetidos à avaliação funcional, $62 \%$ se apre- 
sentavam assintomáticos ou com dor mínima, sem uso de medicação. Não foram avaliados o nível empregatício pré-trauma e as questões litigiosas, que podem ser fatores de confusão nesse tipo de análise. O maior nível de dor encontrado nos pacientes sem fratura do arco posterior foi um achado inesperado, que não conseguimos relacionar a nenhuma outra variável do estudo. Apesar de contemplar um número reduzido de pacientes, nosso levantamento apresentou tempo de seguimento bastante elevado. Não houve correlação estatística entre o resultado clínico e o grau de cifose.

Classicamente, os critérios de indicação cirúrgica para as fraturas vertebrais tipo explosão são cifose superior a $30^{\circ}$, estenose do canal vertebral maior do que $50 \%$, redução da altura do corpo vertebral maior do que $50 \%$ e déficit neurológico ${ }^{(4,17,19-20)}$. Neste estudo, nenhum dos pacientes apresentou algum desses critérios, sendo a fratura da lâmina o único fator que diferenciava os dois grupos estudados. Assim, a fratura dos elementos posteriores não se mostrou um fator que, isoladamente, denote instabilidade da lesão ou ainda que resulte em piores resultados clínico-funcionais.

\section{CONCLUSÃO}

Os pacientes com fratura toracolombar tipo explosão associada à fratura da lâmina não apresentaram resultados clínico e radiográficos inferiores aos do grupo sem e lesão da lâmina, sugerindo que a mesma, isoladamente, não contra-indica o tratamento conservador.

\section{AGRADECIMENTOS}

Agradecemos ao Núcleo de Apoio à Publicação da Faculdade de Ciências Médicas da Santa Casa de São PauloNAP-SC o suporte técnico-científico à publicação deste manuscrito.

\section{REFERÊNCIAS}

1. Holdsworth F. Fractures, dislocations, and fracturedislocations of the spine. J Bone Joint Surg Am. 1970;52(8): 1534-51.

2. Whitesides TE Jr. Traumatic kyphosis of the thoracolumbar spine. Clin Orthop Relat Res. 1977;(128):78-92.

3. Denis F. The three column spine and its significance in the classification of acute thoracolumbar spinal injuries. Spine. 1983;8(8):817-31.
4. McAfee PC, Yuan HA, Lasda NA. The unstable burst fracture. Spine. 1982;7(4):365-73.

5. McAfee PC, Yuan HA, Fredrickson BE, Lubicky JP. The value of computed tomography in thoracolumbar fractures. An analysis of one hundred consecutive cases and a new classification. J Bone Joint Surg Am. 1983;65(4):461-73.

6. Haher TR, Bergman M, O’Brien M, Felmly WT, Choueka J, Welin D, et al. The effect of three columns of the spine on the instantaneous axis of rotation in flexion and extension. Spine. 1991;16(8 Suppl):S312-8.

7. Avanzi O, Chih LY, Meves R. Avaliação do tratamento cirúrgico da fratura toracolombar com material de terceira geração. Rev Bras Ortop. 2002;37(6):226-32.

8. Dickson JH, Harrington PR, Erwin WD. Results of reduction and stabilization of the severely fractured thoracic and lumbar spine. J Bone Joint Surg Am. 1978;60(6):799-805.

9. Aebi M, Etter C, Kehl T, Thalgott J. Stabilization of the lower thoracic and lumbar spine with the internal spinal skeletal fixation system. Indications, techniques, and first results of treatment. Spine. 1987;12(6):544-51.

10. Denis F, Armstrong GW, Searls K, Matta L. Acute thoracolumbar burst fractures in the absence of neurological deficit: a comparison between operative and nonoperative treatment. Clin Orthop Relat Res. 1984;(189):142-9.

11. Bradford DS, Akbarnia BA, Winter RB, Seljeskog EL. Surgical stabilization of fractures and fracture-dislocations of the thoracic spine. Spine. 1977;2(2):185-96.

12. Denis F, Burkus JK. Diagnosis and treatment of cauda equine entrapment in the vertical lamina fracture of lumbar burst fractures. Spine. 1991;16(8 Suppl):S433-9.

13. Cammisa FP Jr, Eismont FJ, Green BA. Dural laceration occurring with burst fractures and associated laminar fractures. J Bone Joint Surg Am. 1989;71(7):1044-52.

14. Eismont FJ, Wiesel SW, Rothman RH. Treatment of dural tears associated with spinal surgery. J Bone Joint Surg Am. 1981; 63(7):1132-6.

15. Wood K, Buttermann G, Mehbod A, Garvey T, Jhanjee R, Sechriest V, Butterman G. Operative compared with nonoperative treatment of a thoracolumbar burst fracture without neurological deficit. A prospective, randomized study. J Bone Joint Surg Am. 2003;85-A(5):773-81. Erratum in: J Bone Joint Surg Am. 2004;86-A(6):1283. Comment in: J Bone Joint Surg Am. 2004;86-A(3):649-50; author reply 650-1. J Bone Joint Surg Am. 2004;86-A(3):651-2; author reply 652.

16. Cantor JB, Lebwohl NH, Garvey T, Eismont FJ. Nonoperative management of stable thoracolumbar burst fracture with early ambulation and bracing. Spine. 1993;18(8):971-6.

17. Chow GH, Nelson BJ, Gebhard JS, Brugman JL, Brown CW, Donaldson DH. Functional outcome of thoracolumbar burst fractures managed with hyperextension casting or bracing and early mobilization. Spine. 1996;21(18):2170-5.

18. de Klerk LW, Fontijne WP, Stijnen T, Braakman R, Tanghe HL, van Linger B. Spontaneous remodeling of the spinal canal after 
conservative management of thoracolumbar burst fractures. Spine. 1998;23(9):1057-60.

19. Mumford J, Weinstein JN, Spratt KF, Goel VK. Thoracolumbar burst fractures. The clinical efficacy and outcome of nonoperative management. Spine. 1993;18(8):955-70.

20. Defino HLA, Fuentes ARR, Remondi PH, Ballim EC. Tratamento conservador das fraturas da coluna toracolombar. Rev Bras Ortop. 2000;35(8):301-8.

21. Aligizakis A, Katonis P, Stergiopoulos K, Galanakis I, Karabekios S, Hadjipavlou A. Functional outcome of burst fractures of the thoracolumbar spine managed non-operatively, with early ambulation, evaluated using the load sharing classification. Acta Orthop Belg. 2002;68(3):279-87.

22. Magerl F, Aebi M, Gertzbein SD, Harms J, Nazarian S. A comprehensive classification of thoracic and lumbar injuries. Eur Spine J. 1994;3(4):184-201.

23. Kuklo TR, Polly DW, Owens BD, Zeidman SM, Chang AS, Klemme WR. Measurement of thoracic and lumbar fracture kyphosis: evaluation of intraobserver, interobserver, and technique variability. Spine. 2001;26(1):61-5; discussion 66.

24. Avanzi O, Chih LY, Meves R, Caffaro MFS, Miura MT. Avaliação tomográfica do fragmento retropulsado nas fraturas toracolombares tipo explosão. Coluna/Columna. 2006;5(4):247-52.

25. Ware JE Jr, Sherbourne CD. The MOS 36-item short-form health survey (SF-36). I. Conceptual framework and item selection. Med Care. 1992;30(6):473-81.

26. Frankel HL, Hancock DO, Hyslop G, Melzak J, Michaelis LS, Ungar GH, et al. The value of postural reduction in the initial management of closed injuries of the spine with paraplegia and tetraplegia. I Paraplegia. 1969;7(3):179-92.

27. Avanzi O, Chih LY, Meves R. Avaliação do tratamento cirúrgico tardio na fratura toracolombar tipo explosão. Coluna/Columna 2002;1(2):88-93.

28. Tropiano P, Huang RC, Louis CA, Poitout DG, Louis RP. Functional and radiographic outcome of thoracolumbar and lumbar burst fractures managed by closed orthopaedic reduction and casting. Spine. 2003;28(21):2459-65.

Declaração de inexistência de conflitos de interesse: Declaramos para os devidos fins que não há qualquer conflito de interesse que impeça a publicação deste artigo. 